\title{
An analysis of identifiable risk factors for obesity and diabetes in American adults
}

\author{
Damien Byas ${ }^{1 *}$ and Rashmi Dixit ${ }^{2}$ \\ ${ }^{1}$ Center for Healthcare and Organizational Research, USA \\ ${ }^{2}$ Walnut Creek Medical Center, CA, USA
}

\begin{abstract}
Obesity is postulated as a risk factor for several chronic conditions such as diabetes mellitus and cardiovascular disease, gallstones, gastro-esophageal reflux, musculoskeletal diseases such as osteoarthritis and gout, sleep apnea, and even certain. Between 2015 and 2016, 39\% of American Adults were diagnosed as obese and more than 93 million adults in the U.S. were significantly affected by this growing trend. Factors often associated with genetics, modifiable behaviors, and lifestyle patterns, have been found to attribute to the health status of many American children and adults. More extensive research may be needed to examine specific identifiable risk factors which may be associated with disease and other negative health outcomes in the United States. Community members, medical professionals, and key stakeholders across American communities are in the process of uniting efforts to educate community members to better promote health educational awareness and disease prevention to focus more on reducing prevalence and mortality rates of preventable diseases and poor health conditions.
\end{abstract}

\section{Introduction}

Between 2015 and 2016, 39\% of American Adults were diagnosed as obese and more than 93 million adults in the U.S. were significantly affected by this growing trend [1]. The prevalence of obesity was higher among middle-aged adults (42.8\%) than among younger adults (35.7\%) [1]. An examination of potential risk factors which may be associated with the growth in adult obesity prevalence rates in America may be warranted as Obesity remains as one of the more serious or preventable diseases which often affect Americans in large proportions. As more research on obesity prevalence is conducted, there may also be more research initiatives applied to address negative health concerns which may both impact and be impacted by obesity rates in the U.S.

Research has found that obesity outcomes may be a related problem for a person who may have been diagnosed with diabetes, coronary heart disease, or snoring and other research has found that type 2 diabetes may be associated with an increase in visceral fat mass (ie abdominal fat) as opposed to general adiposity, and this is an outcome almost often associated with obesity $[2,3]$. Researchers at the Mexico Bariatric Center found that Obesity increases one's risk of diabetes, some cancers, heart disease, stroke, arthritis, gallstones, breathing problems, hypertension, and higher healthcare costs. It was also reported that obesity may only limit one's agility and fundamental human abilities, but obesity may also serve as a gateway for other medical and health concerns such as diabetes, hypertension, certain forms of cancer, and even death [4]. Because of these types of health outcomes, a more thorough analysis of the potential relationship between obesity prevalence and diabetes diagnosis among American adult populations may be needed. Such a research initiative should also include an examination of other possible factors or health concerns which may be related to both obesity and diabetes outcomes.

\section{Purpose}

The purpose of this research was to investigate potential identifiable risk factors which may be associated with obesity and diabetes diagnosis in American adults $(N=3,045,180)$ [5]. The age of the sample group for this research study included American adults 20 years and older. The 2010 U.S. Census data supported the notion that the sample adequately represented the population of U.S. adult citizens. The researchers were hopeful that the results of this research study would contribute to initiatives to educate Americans on potential strategies and approaches which may assist in reducing obesity and diabetes rates across communities.

There appears to be unsubstantiated research evidence which clearly identifies all possible behavioral, personal, and environmental factors which may attributes to diabetes and obesity diagnosis and other associated negative health outcomes. In addition to the limited amount of research which has investigated these three key factors, mental, social, and possible holistic health related issues may also serve as indicators which may attribute to health outcomes as well.

\section{Method}

A random sample $(N=3,045,180)$ of adult Americans was analyzed for this research study. A multiple linear regression model was applied to determine if five health indicators were found to be significant predictors for obesity $(a=0.05)$. The four health indicators which were examined in this research study were Hypertension, Diabetes, Drug Abuse, and Alcohol Abuse. In addition to the application of the regression model, a Pearson $r$ analysis was conducted to examine the possible strength of the relationship between obesity and the four health indicators. A Pearson $r$ analysis was also applied to determine

${ }^{*}$ Correspondence to: Damien Byas, Center for Healthcare and Organizational Research, USA,

Key words: diabetes, obesity, cardiovascular, health, disease

Received: August 30, 2018; Accepted: September 29, 2018; Published: October 06,2018 
the strength of the relationship between Diabetes and four health determinants, to include obesity diagnosis. The NIS data examined in this research study were collected from American hospitals in which 46 U.S. states were cooperative participants in providing state health related data [5].

\section{Results}

The findings from this research study provided evidence of high disease prevalence rates for each of the examined health outcomes (Table 1.). The Pearson $r$ analysis results revealed very strong significant relationships between obesity and the health outcomes of diabetes, substance abuse, and drug abuse (Table 2). The second Pearson $r$ analysis produced very strong significant relationships between diabetes and disease outcomes of hypertension, obesity, substance abuse, and drug abuse (Table 3 ). In addition to these results from the Pearson $r$ analyses, the results of this research study also verified a statistically significant multiple regression model $(F=12.663, p<0.01)$ (Table 4$)$ and found that health outcomes of hypertension, diabetes, alcohol abuse, and drug abuse, were each significant predictors of obesity in American adults (Table 5).

\section{Discussion}

Based on the results of this study, the researchers have concluded that specific attention should be given to address the importance of the implementation of a healthy diet and lifestyle to address the health outcomes for diabetes and obesity as well as the associated identifiable risk factors examined in this research study. One important note to

Table 1. Disease Prevalence Rates for American Adults $(a=0.05)$

\begin{tabular}{|c|c|}
\hline & $\boldsymbol{n}$ \\
\hline Hypertension & 509,695 \\
\hline Diabetes & $1,124,619$ \\
\hline Obesity & 801,196 \\
\hline Drug Abuse & 312,860 \\
\hline Alcohol Abuse & 296,810 \\
\hline
\end{tabular}

Table 2. Pearson R Correlation: Diabetes

\begin{tabular}{|c|c|c|}
\hline & Diabetes & P value \\
\hline Hypertension & 0.860 & 0.031 \\
\hline Obesity & 0.868 & 0.028 \\
\hline Drug Abuse & 0.995 & 0.000 \\
\hline Alcohol Abuse & 0.928 & 0.011 \\
\hline
\end{tabular}

Table 3. Pearson R Correlation: Obesity

\begin{tabular}{|c|c|c|}
\hline & Obesity & P value \\
\hline Hypertension & 0.720 & 0.085 \\
\hline Diabetes & 0.868 & 0.028 \\
\hline Drug Abuse & 0.899 & 0.019 \\
\hline Alcohol Abuse & 0.862 & 0.030 \\
\hline
\end{tabular}

Table 4. ANOVA

\begin{tabular}{|c|c|}
\hline F & P value \\
\hline $\mathbf{1 2 . 6 6 3}$ & 0.038 \\
\hline
\end{tabular}

Table 5. Multiple Regression Coefficients

\begin{tabular}{|c|c|c|}
\hline & B & P value \\
\hline Hypertension & 2.906 & 0.000 \\
\hline Diabetes & 0.257 & 0.000 \\
\hline Alcohol Abuse & 13.857 & 0.000 \\
\hline Drug Abuse & 0.887 & 0.035 \\
\hline
\end{tabular}

DV: Obesity

make based on the findings which were assessed between the factors of alcohol abuse and drug abuse, is that both factors exist as a result of unhealthy behavior and engagement in such practices could be highly reduced through the implementation of behavioral health modification. Reducing both unhealthy and risk behaviors could be a critical step in addressing effective disease prevention strategies and in reducing disease outcome and mortality rates across communities.

\section{References}

1. Hales CM, Carroll M, Fryar CD, Ogden CL (2016) Prevalence of Obesity Among Adults and Youth: United States, 2015-2016. NCHS Data Brief No. 288, October 2017.

2. NICE (2014) Obesity: identification assessment and management of overweight and obesity in children young people and adults; NICE Clinical Guideline (November 2014)

3. Neeland IJ, Turer AT, Ayers CR, Powell-Wiley TM, Vega GL, et al. (2012) Dysfunctional adiposity and the risk of prediabetes and type 2 diabetes in obese adults. JAMA 19308: 1150-1159. [Crossref]

4. Mexico Bariatric Center (2018). Obesity Statistics United States and Across the Globe: Quick Overview of Obesity. Retrieved from: https://mexicobariatriccenter.com/ obesity-statistics-update-usa-world/

5. National Inpatient Sample Database (NIS) (2017). Agency for Healthcare Research and Quality, Rockville, MD.

Copyright: (C2018 Byas D. This is an open-access article distributed under the terms of the Creative Commons Attribution License, which permits unrestricted use, distribution, and reproduction in any medium, provided the original author and source are credited. 